\title{
Les filtres Beaudrey : un créneau ouvert sur le monde
}

\author{
P.E. Jackson
}

Beaudrey

La société E. Beaudrey \& Cie (45 personnes) est spécialisée exclusivement dans le tamisage mécanique de l'eau depuis sa création en 1912.

Cette activité se subdivise en deux champs d'activité : le tamisage des prises d'eau à plan d'eau libre et le tamisage sur conduite. Les utilisateurs en sont les centrales thermiques et nucléaires, les terminaux émetteurs et récepteurs de GNL (gaz naturel liquéfié) ainsi que toutes les industries ayant à échanger de grandes quantités de calories avec le milieu extérieur.

De toute époque, les centrales électriques françaises ont été en avance dans ce domaine sur leurs homologues étrangères tant par la taille unitaire des machines que par les exigences en matière de service rendu et de fiabilité. Les exigences du marché national ont conduit Beaudrey à développer des solutions originales pour faire face à tous les cas que la nature et la civilisation proposent en matière de débris à éliminer avant pompage. La société dispose en fait de deux gammes de machines : l'une classique capable de satisfaire aux appels d'offres selon les standards européens, américains ou japonais, l'autre destinée à résoudre des difficultés particulières. Les matériels sont sans équivalent ailleurs.

Quelques exemples de par le monde:

Usine de gaz naturel liquéfié de Bintulu, Malaisie

Cette usine pompe $64 \mathrm{~m}^{3} / \mathrm{s}$ d'une eau de mer susceptible de contenir des quantités énormes de grosses méduses (48 tonnes/heure). Quatre tambours filtrants spéciaux, surdimensionnés ( $12 \mathrm{~m}$ de diamètre, $6 \mathrm{~m}$ de large) à grande vitesse de rotation, avec lavage à haute pression et élément filtrant spécial évitant l'adhérence des méduses, fonctionnent sans défaillance depuis douze ans.

\section{Protection de la vie aquatique aux Etats-Unis}

Certaines zones des Etats-Unis mettent l'accent sur la protection des poissons (grands lacs, etc) et imposent des contraintes de taux de survie des poissons capturés sur les tamis telles que seul le système « Scoop-a-fish » développé pour certaines centrales du programme nucléaire français peut y satisfaire. Ce système Beaudrey est maintenant imposé par voie réglementaire dans les cas les plus sensibles.

\section{Palo Seco - Puerto Rico}

A Puerto Rico, la centrale thermique de Palo Seco en service depuis plusieurs années, était arrêtée environ
180 jours par an par la défaillance du tamisage de l'eau de refroidissement, aucun des nombreux types de filtre à chaînes et dégrilleurs disponibles sur le marché américain n'ayant pu faire face aux arrivées d'algues dans la prise d'eau.

Après essais pendant un an d'un filtre à chaîne spécial Beaudrey à très grande vitesse, à nettoyage renforcé utilisant des composants spécialement développés et une nouvelle approche structurelle, le problème a été résolu et la totalité des filtres du parc de centrales porto-ricaines renouvelé avec ce type de filtre (19 machines).

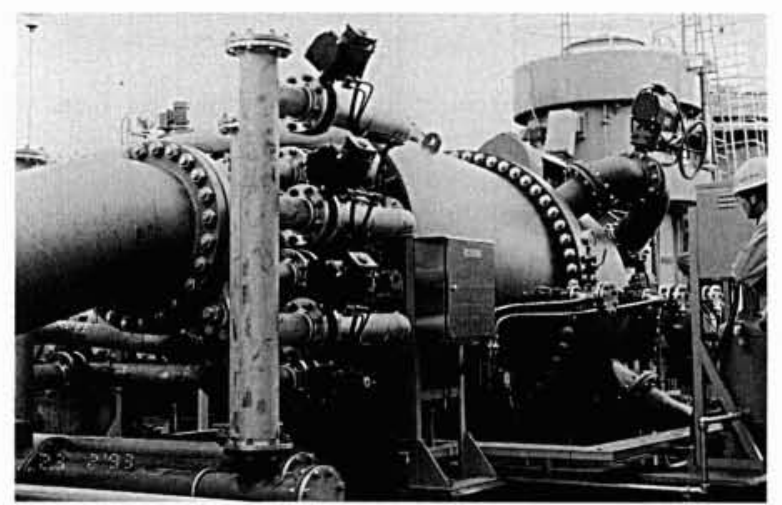

1. Filtre installé à la centrale d'Higashi Ohgishima.

Le terminal GNL d'Higashi Ohgishima comporte un circuit d'incendie très important $\left(4 \times 3500 \mathrm{~m}^{3} / \mathrm{h}\right)$. Lors de la mise en route des pompes, l'afflux de débris (coquillages, algues, plastiques) est tel que les filtres sur conduite (japonais d'origine) - pourtant largement dimensionnés éclataient sous la perte de charge au bout de quarante secondes au plus.

Des filtres conçus par Beaudrey, combinant deux étapes de traitement $(10$ et $0.8 \mathrm{~mm}$ ) ont totalement résolu le problème. Ce type de filtre est maintenant standardisé par TEPCO pour ses prochaines réalisations.

En Thaïlande, les eaux d'estuaires sont chargées en fibres fines qui s'enroulent sur les toiles filtrantes créant un 




2. Centrale de Bang Pakong (Thailande).

feutrage qui finit en obstruction totale. Il n'existe alors qu'un remède : arrêter le filtre et brûler la couche de feutre à la torche.
Nous avons développé un panneau filtrant appelé « Nocling " à forte épaisseur et profilé hydrauliquement pour éviter les tourbillons propices aux enroulements fibreux. Les filtres ainsi équipés sont les seuls à ne nécessiter aucun arrêt pour entretien (les centrales de Bank Pakong et South Bangkok en sont maintenant équipées).

\section{Centrale de Cattenom - France}

Conjointement avec la société Technos, nous venons de terminer les essais d'endurance (trois ans) d'un système de protection et de nettoyage en continu des échangeurs à plaques, éliminant ainsi la principale vulnérabilité de cet échangeur, si avantageux par ailleurs.

Equipant deux tranches nucléaires françaises, ce système suscite un vif intérêt à l'étranger.

Ainsi, par des réalisations très originales résolvant des difficultés auparavant sans solution, la notoriété de la société Beaudrey s'étend et lui permet de pénétrer sur des marchés auparavant inaccessibles. 